\title{
Estimación de fugas en un sistema industrial real mediante modelado por señales aditivas
}

\author{
Ester Sales-Setién, David Tena, Ignacio Peñarrocha \\ Departamento de Ingeniería de Sistemas Industriales y Diseño \\ Universitat Jaume I, Castellón, España \\ \{esales,david.tenatena,ipenarro\}@uji.es
}

\section{Resumen}

Este trabajo aborda el problema de estimación de fugas en una red industrial real de tuberías y tanques. Para lograr este objetivo, se propone el uso de un modelo basado en el principio de conservación de la masa en el que se incluyen las fugas como señales aditivas. A continuación, se plantean observadores tipo PI para la estimación de estas fugas. El trabajo incluye un análisis de las prestaciones del observador en relación a la estimación de las fugas y al diagnóstico de las mismas. También se estudia el efecto del comportamiento no Gaussiano del ruido que afecta a las mediciones reales.

Palabras clave: Fugas, aplicación industrial, estimación, diagnóstico de fallos, observadores PI.

\section{Introducción}

Las redes de tuberías y tanques representan importantes activos en la industria de procesos. Con independencia del esfuerzo dedicado al diseño y construcción de estas redes, las tuberías son propensas a sufrir fugas debido a la corrosión, la erosión o defectos de materiales entre otros. En muchos casos, el daño causado por las fugas se extiende más allá de los costes monetarios ya que las pérdidas de producto pueden ser peligrosas para los seres humanos y el medio ambiente. Así, la comunidad científica ha dedicado mucho esfuerzo al estudio de este problema. Los trabajos pioneros en el campo versaban sobre la rehabilitación y el remplazamiento de tuberías, ver [1]. En las últimas décadas, sin embargo, las técnicas de detección de fugas han acaparado más atención, ver [2].

La clasificación incluida en [3] divide las técnicas de detección de fugas en dos grandes grupos. En primer lugar, existen las técnicas directas o externas, las cuales requieren el uso de hardware especializado como sensores ópticos o de inspección del suelo. En segundo lugar, se tienen las técnicas indirectas o internas que no requieren el uso de sensores especializados y se basan en la información proporcionada por las mediciones del proceso. Entre ellas, [4] distingue entre las estrategias que se basan en el principio de conservación de la masa y los métodos que usan modelos analíticos de la dinámica del fluido en el interior de la tubería. Muchas contribuciones como [5] trabajan con estos métodos para la identificación de fugas; sin embargo, estas estrategias están basadas en complejos modelos que, la mayoría de las veces, son muy difíciles de calibrar y requieren de personal especializado para su gestión, [6]. Además, muchas redes industriales no están provistas de transductores de presión y la aplicación de estas estrategias resulta imposible. Por su parte, los métodos basados en el principio de conservación de la masa, como los presentados en [7], son fáciles de usar y pueden ser implementados fácilmente ya que utilizan mediciones proporcionadas por instrumentación presente en toda red industrial, [8].

En la bibliografía encontramos múltiples trabajos que usan técnicas de identificación basadas en observadores para la detección de fugas, por ejemplo [9]. A excepción de contadas contribuciones, i.e. [10], la mayoría de estos trabajos no usan datos reales y, en muchos casos, se asumen condiciones ideales de ruido. Los autores de [11] señalan la necesidad de estudios más detallados y comprensibles que permitan mejorar la efectividad y factibilidad del diagnóstico de fugas en redes de ambientes industriales. El principal objetivo de este trabajo es la aplicación de estimadores de fugas a una red industrial real de tanques y tuberías. Se utilizarán técnicas aditivas basadas en modelo que trabajan con el principio de conservación de la masa.

La organización de este trabajo es la siguiente. Primero, se establece el problema en la Sección 2, donde se incluye el modelo de la red de tanques y tuberías con sus sensores. En la Sección 3, se añaden las fugas al modelo anterior y se presentan observadores tipo PI (Proporcional-Integral) para la estimación de las mismas. En la Sección 4, se estudia la aplicación de técnicas de diagnóstico de fallos basadas en las señales de fuga estimadas. Finalmente, en la Sección 5, aplicamos la metodología propuesta a una red industrial real y, en la Sección 6, resumimos las principales conclusiones 


\section{Descripción del problema}

Considérese un conjunto de $i=\left\{1, \ldots, n_{v}\right\}$ tanques de almacenamiento de fluidos interconectados a través de una red de $j=\left\{1, \ldots, n_{q}\right\}$ tuberías. Para modelar esta red, se propone la aplicación del balance de masas a cada uno de los tanques $i$ de la red, i.e.,

$$
\dot{M}_{i}=\Sigma_{j=1}^{n_{q}} c_{i, j} m_{j}
$$

donde $m_{j}$ es el caudal másico transportado por la tubería $j, \dot{M}_{i}$ es la derivada de la masa de fluido contenido en el tanque $i$ y $c_{i, j}$ es una constante que describe la relación entre el caudal de la tubería $j$ y la variación experimentada por el tanque $i$. Así, $c_{i, j}=0$ si la tubería no está conectada al tanque y $c_{i, j}=\{-1,1\}$ dependiendo de si la tubería extrae o inyecta fluido en él.

Debido al elevado coste de los caudalímetros másicos como los medidores de Coriolis, los caudales másicos no se miden, en general, con estos instrumentos en la industria. Por el contrario, es mucho más común encontrar caudalímetros volumétricos magnéticos o de diferencial de presión. Éstos se calibran con una densidad predefinida del producto, $\rho_{j}^{0}$, dando el siguiente caudal másico:

$$
m_{j}^{0}=\rho_{j}^{0} m_{j}
$$

Las condiciones ambientales, entre otros, pueden afectar a la densidad del producto por lo que la densidad real del producto, $\rho_{j}$, no cumple más la relación $\rho_{j}=\rho_{j}^{0}$. Así, modelamos, los caudales másicos proporcionados por los sensores volumétricos, denotados con $u_{j}$, como

$$
u_{j}=m_{j}+\eta_{j}
$$

donde $\eta_{j}$ no solo tiene en cuenta los ruidos de medida sino que también considera el sesgo que se producen por la diferencia entre la densidad de referencia del producto, $\rho_{j}^{0}$, y la densidad real, $\rho_{j}$, i.e. $m_{j}-m_{j}^{0}$.

Lo mismo se aplica a la masa del fluido contenido en los tanques. En la industria, la masa se calcula a partir de las medidas proporcionadas por sensores de nivel, las dimensiones del tanque y la densidad de referencia del producto, $\rho_{j}^{0}$, i.e.

$$
M_{i}^{0}=\rho_{i}^{0} S_{i} h_{i}
$$

con $S_{i}$ la sección equivalente del tanque $i$ y $h_{i}$ la altura equivalente medida por los sensores. De nuevo, modelamos las medidas másicas, a las que llamamos $s_{i}$, como

$$
s_{i}=M_{i}+\nu_{i}
$$

donde $\nu_{i}$ recoge los ruidos de medida, los sesgos de densidad y la incertidumbre en las dimensiones del tanque.

La red anterior puede quedar afectada por fugas en sus tuberías, las cuales corromperían el sistema. Si tiene lugar una fuga, la ecuación (1) no se cumple más. En la siguiente sección mostramos como modificar la relación (1) para que ésta tenga en cuenta las posibles fugas en las tuberías.

El objetivo principal de este trabajo es proporcionar un algoritmo de estimación de fugas que utilice la información proporcionada por las mediciones $u_{j}$ y $s_{i}$. También se tratará de caracterizar las prestaciones de este estimador para construir un diagnosticador de fallos que diferencie entre la presencia y ausencia de fugas en el sistema. En la literatura de diagnóstico de fallos, es común considerar que los ruidos que afectan a las mediciones son Gaussianos. Las mediciones reales, sin embargo, suelen estar afectadas por desviaciones no Gaussianas como sesgos, i.e. (3) y (5). Otras discrepancias con respecto a esta suposición derivan de otras causas como el uso de filtros en sensores inteligentes o temas de cuantización, que dependen de la resolución digital. Con todo, en el trabajo se tratará de discutir cuán críticos son estos comportamientos para el diagnóstico de fallos.

Se desea que los algoritmos de este trabajo se puedan implementar en hardware industrial como PLCs o Sistemas de Control Distribuido. Por ello, a lo largo del artículo, se trabajará en el espacio discreto con un periodo de muestro $T_{s}$. También se estudiará el efecto de este periodo de muestro en la no Gaussianidad de los ruidos que afectan a las mediaciones.

\section{Estimación de Fugas}

\subsection{Modelado Aditivo de las Fugas}

Para tener en cuenta las posibles fugas en la red de tanques y tuberías, la ecuación (1) puede ser rescrita como

$$
\dot{M}_{i}=\Sigma_{j=1}^{n_{q}} c_{i, j}\left(m_{j}+l_{i, j}\right),
$$

donde $l_{i, j}$ representa el caudal másico fugado en la tubería $j$ conectada al tanque $i$. De acuerdo con lo expuesto en [12], para que las señales de fallo sean identificables, éstas deben ser también detectables e aislables. Un fallo es detectable si sus variaciones afectan a las salidas medibles del sistema. Así, un fallo de fuga $l_{i, j}$ es detectable con $M_{i}$ si $c_{i, j} \neq 0$. Por otra parte, un fallo aditivo como $l_{i, j}$ solo es aislable si el número total de fallos que puede afectar al sistema no es superior al número de salidas medibles. Dado que una única medida de salida 
$M_{i}$ está disponible por tanque, solo se puede llegar a estimar el caudal másico total fugado en el conjunto de las tuberías conectadas a cada tanque $i$, i.e

$$
M_{i}=\Sigma_{j=1}^{n_{q}} c_{i, j} m_{j}+f_{i},
$$

con $f_{i}=\Sigma_{l=1}^{n_{q}} c_{i, j} l_{i, j}$. Finalmente, definimos la función de transferencia entre un fallo $f_{i}$ y la salida del sistema como $G_{f_{i}}(s)$. Para que un fallo $f_{i}$ sea identificable se necesita que la inversa de $G_{f_{i}}(s)$ sea estable y causal. Como la salida del sistema es $M_{i}$, tenemos que $G_{f_{i}}^{-1}(s)=s$, lo que significa que $f_{i}$ no es identificable a partir de $M_{i}$. Para solucionar este problema, proponemos utilizar la derivada de $M_{i}$, que denotamos como $\dot{M}_{i}$, como salida del sistema; así, $G_{f_{i}}^{-1}(s)=1$, i.e. $f_{i}$ es identificable a partir de $\dot{M}_{i}$. La discretización del modelo del sistema que permite la estimación de las fugas es

$$
y_{i}[k]=E_{i} u[k]+E_{i} \eta[k]+\mu_{i}[k]+f_{i}[k],
$$

con

$$
\begin{aligned}
E_{i} & =\left[\begin{array}{lll}
c_{i, 1} & \ldots & c_{i, n_{q}}
\end{array}\right], \\
u[k] & =\left[\begin{array}{lll}
u_{1}[k] & \ldots & u_{n_{q}}[k]
\end{array}\right]^{T}, \\
\eta[k] & =\left[\begin{array}{lll}
-\eta_{1}[k] & \ldots & -\eta_{n_{q}}[k]
\end{array}\right]^{T},
\end{aligned}
$$

y donde $\mu_{i}[k]$ es el ruido de la nueva medición de salida $y_{i}[k]$, i.e.

$$
\begin{aligned}
& y_{i}[k]=\frac{1}{T_{s}}\left(s_{i}[k]-s_{i}[k-1]\right), \\
& \mu_{i}[k]=\frac{1}{T_{s}}\left(\nu_{i}[k]-\nu_{i}[k-1]\right) .
\end{aligned}
$$

\subsection{Algoritmo de Estimación}

Para favorecer la precisión del estimador en estado estacionario, proponemos el uso de observadores tipo PI. Así, modelamos la dinámica de los fallos $f_{i}$ de $(8)$ como

$$
f_{i}[k+1]=f_{i}[k]+\Delta f_{i}[k],
$$

donde $\Delta f_{i}[k]$ representa la variación de las señales de fallo $f_{i}$ entre dos instantes consecutivos. La ecuación (11) permite modelar, por ejemplo, señales tipo escalón $(\Delta f[k]$ sólo tiene un valor no nulo en el momento de aparición del fallo) o señales tipo rampa $(\Delta f[k]$ toma un valor constante). Este tipo de modelo de fallos ha sido extensamente utilizado en la bibliografía para analizar el comportamiento de detectores de fallos, ver $[13,14]$.

El observador PI propuesto para la estimación del fallo $f_{i}$, que denotamos como $\hat{f}_{i}$, es

$$
\hat{f}_{i}[k+1]=\hat{f}_{i}[k]+l_{i}\left(y_{i}[k]-\hat{f}_{i}[k]-E_{i} u[k]\right),
$$

donde $l_{i}$ es la ganancia que actualiza las predicciones obtenidas del modelo con las mediciones.

\subsection{Diseño Basado en Prestaciones}

Primero, definamos el error de estimación del fallo como

$$
\tilde{f}_{i}[k]=f_{i}[k]-\hat{f}_{i}[k] .
$$

Su dinámica viene dada por

$$
\begin{gathered}
\tilde{f}_{i}[k+1]=\left(1-l_{i}\right) \tilde{f}_{i}[k]+\Delta f_{i}[k]- \\
l_{i}\left(E_{i} v_{i}[k]+\mu_{i}[k]\right) .
\end{gathered}
$$

De (14) se deduce que $l_{i}$ fija los polos de la dinámica de $\tilde{f}_{i}$. Para un sistema de primer orden, una medida estandarizada para la caracterización de la velocidad de respuesta es la constante de tiempo $\tau_{i}$ definida como

$$
\tau_{i}=-T_{s} / \ln \left(1-l_{i}\right) .
$$

Esta constante fija, entre otros, el tiempo de establecimiento al $98 \%$ frente a fallos escalón como $t_{s, 98 \%}=4 \tau$. También podemos utilizar otros índices como el Error Cuadrático Acumulado (ISE, del inglés Integral Squared Error) del error que, cuando ocurre un fallo tipo escalón, viene dado por

$$
\mathrm{ISE}_{i}=T_{s}\left(1-\left(1-l_{i}\right)^{2}\right) \text {. }
$$

Segundo, denominamos a la varianza del error $\tilde{f}_{i}[k]$ como $\sigma_{i}^{2}$. Para calcular $\sigma_{i}^{2}$ debemos tener en cuenta la correlación entre $\nu_{i}[k]$ y $\nu_{i}[k-1]$ dentro de $\mu_{i}[k]$, ver (10). Así, en estado estacionario y cuando ningún fallo está presente en el sistema, $\sigma_{i}^{2}$ satisfice la siguiente ecuación de Lyapunov:

$$
\begin{aligned}
\mathcal{F}_{i} & =A_{i} \mathcal{F}_{i} A_{i}^{T}+B_{i} \mathcal{V}_{i} B_{i}^{T}, \\
\sigma_{i}^{2} & =R \mathcal{F}_{i} R^{T},
\end{aligned}
$$

$\operatorname{con} R=\left[\begin{array}{ll}1 & 0\end{array}\right]$,

$$
A_{i}=\left[\begin{array}{cc}
l_{i} / T_{s} & 1-l_{i} \\
0 & 0
\end{array}\right], B_{i}=\left[\begin{array}{cc}
-l_{i} E_{i} & -l_{i} / T_{s} \\
0 & 1
\end{array}\right]
$$

y $\mathcal{V}_{i}$ la covarianza del vector $v_{i}[k]=\left[\eta[k] \quad \nu_{i}[k]\right]$, i.e. $\mathcal{V}_{i}=\mathbf{E}\left\{v_{i}[k] v_{i}^{T}[k]\right\}$. La matriz $\mathcal{F}_{i}$ da la varianza del vector $\left[f_{i}[k]^{T} \quad \nu_{i}[k]^{T}\right]^{T}$.

Las ecuaciones (15)-(16) y (17) muestran que hay un compromiso entre la habilidad de seguimiento del estimador y la precisión de las estimaciones. Para diseñar el estimador de fallos, se puede fijar el comportamiento temporal de las estimaciones con (15) o (16) y obtener la precisión de las estimaciones con (17) o seguir el procedimiento inverso, i.e., fijar la precisión con (17) y caracterizar la habilidad de seguimiento con (15)-(16). 


\section{Diagnóstico de Fallos}

Dadas las características del estimador presentado anteriormente en (12), proponemos usarlo para el diagnóstico de fugas. Así, se introduce la siguiente decisión

$$
\begin{cases}\text { if }\left|\hat{f}_{i}[k]\right| \geq f_{m, i} & \text { Fuga } f_{i} \\ \text { otherwise } & \text { No fuga } f_{i}\end{cases}
$$

donde $f_{m, i}$ denota el mínimo fallo diagnosticable $f_{i}$, es decir, $f_{m, i}$ es el valor constante de fallo que hace saltar la alarma de fallo $f_{i}$ si se tiene en cuenta que no hay otros fallos ni ruidos en el sistema.

$$
f_{m, i}=\left\{\left|f_{i}[k]\right|: \begin{array}{l}
f_{i}[k]=f_{i}[k-1], \forall k \\
\operatorname{lím}_{k \rightarrow \infty}\left|\hat{f}_{i}[k]\right| \geq f_{m, i}
\end{array}\right\} .
$$

Se define ahora el ratio de falsas alarmas (FAR, del inglés False Alarm Rate), que denotamos con $\phi_{i}$, como la probabilidad de hacer saltar una falsa alarma, es decir,

$$
\left.\phi_{i}=\operatorname{Pr}\left\{\left|\hat{f}_{i}[k]\right| \geq f_{m, i}\right): f_{i}[k]=0\right\} .
$$

Si asumimos que los ruidos que afectan al sistema son Gaussianos, tenemos que el error de estimación asociado a cada estimación del fallo, $\tilde{f}_{i}[k]$, tiene una distribución normal de media cero. Entonces, $f_{m, i}$ debería satisfacer la siguiente relación para garantizar cierto FAR:

$$
f_{m, i}=\Phi^{-1}\left(1-\phi_{i} / 2\right) \sigma_{i}
$$

donde $\Phi^{-1}$ es la inversa de la función de densidad de una variable aleatoria normal. Esto significa que el mínimo fallo diagnosticable puede verse también como el cuantil utilizado para construir el intervalo de confianza de nivel $1-\phi(i) / 2$.

Nota 1 Si los ruidos tienen sesgo, debemos incrementar el umbral $f_{m, i}$ en una cantidad igual a $\sum_{j}\left|F_{i, j}\right| \bar{v}_{i, j}$ donde $F_{i, j}$ es la ganancia estática del ruido del sensor $v_{i, j}$ al fallo estimado $\hat{f}_{i}, y \bar{v}_{i, j}$ es el sesgo en el sensor $v_{i, j}$.

\section{Aplicación}

Considérese la red mostrada en la Figura 1. Hay un tanque de almacenamiento de materia prima conectado a un tanque de alimentación. Se denota la masa en esos tanques como $M_{1}$ y $M_{2}$. La máxima capacidad de los tanques es, respectivamente, 10000 y 800 toneladas métricas. Estos tanques se conectan a través de una tubería que proporciona un caudal másico $m_{1}$ de hasta $25 \mathrm{t} / \mathrm{h}\left(c_{1,1}=-1\right.$ y $\left.c_{2,1}=1\right)$. El tanque de alimentación tiene dos consumidores, cuyos caudales másicos se denotan como $m_{2}$ y $m_{3}\left(c_{2,2}=c_{2,3}=-1\right)$. Estos caudales másico son de hasta 15 y 10 t/h respectivamente. Todas las tuberías pueden sufrir una fuga (i.e., las fugas $l_{1,1}$ y $\left.l_{2,1 / 2 / 3}\right)$. En este análisis, nos enfocamos en la detección de la fuga $l_{2,1}$ ya que, en realidad, esta tubería es la más larga y la menos accesible para los operarios. Aplicamos la técnica de estimación de fallo en dos situaciones, una con datos simulados y otra con datos reales de industria en los que hemos añadido una fuga simulada. Para $T_{s}=1$, las señales reales necesarias para la

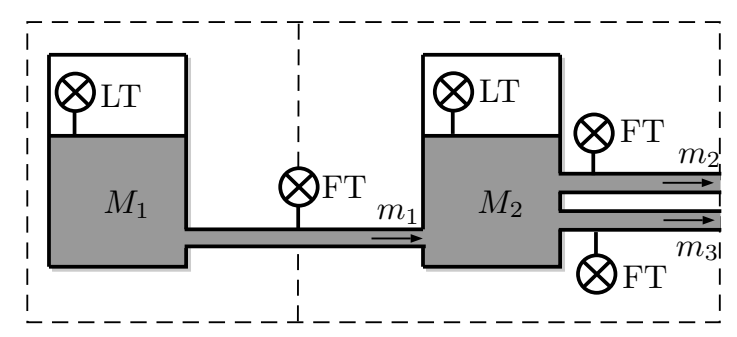

Figura 1: Arquitectura de la red de tanques, tuberías y transductores.

estimación son las que se muestran en la Figura 2. La Figura 3 muestra que la cuantización en las mediciones $s[k]$ afecta a $y_{2}[k]$ cuando se calcula con $T_{s}=1 \mathrm{~s}$; su ruido está correlado. Si se utiliza $T_{s}=20 \mathrm{~s}$, la correlación desaparece con el coste de un retardo mínimo en la detección de 20 segundos. Este retardo es despreciable para el caso de estudio; por lo tanto, elegimos $T_{s}=20 \mathrm{~s}$ para la implementación. En este caso, la desviación estándar del ruido de medición de $y_{2}[k]$ es 79,2 t/h. En el peor de los casos, la desviación estándar de cada uno de los sensores $u_{1}[k], u_{2}[k]$ y $u_{3}[k]$ es 0,053 $\mathrm{t} / \mathrm{h}, 0,0026 \mathrm{t} / \mathrm{h}$ y $0,022 \mathrm{t} / \mathrm{h}$, respectivamente. Vemos que el reto principal del problema reside en la diferencia del orden de magnitud del ruido entre las mediciones del tanque y las que vienen de las tuberías.

Se han diseñado diferentes observadores para el estimador (12) con diferentes constantes de tiempo $\tau_{2}$ desde 20 min a 180 min y con diferentes FARs $\left(\phi(1)=10^{-3}, \phi(2)=10^{-6}\right.$ y $\left.\phi(3)=10^{-9}\right)$. La parte superior de la Figura 4 muestra el mínimo fallo detectable que corresponde a cada diseño. Se ve que para un FAR dado, el mínimo fallo detectable $f_{m, 2}$ disminuye cuando se incrementa la constante de tiempo del estimador. Conseguir gran sensibilidad a los fallos (es decir, un $f_{m, 2}$ pequeño) requiere una mayor constante de tiempo del estimador si uno quiere asegurar a su vez ratios de falsas alarmas pequeños.

Bajo la presencia de un fallo $f_{2}$ tipo escalón, la estimación $\hat{f}_{2}[k]$ se comporta como un sistema de primer orden con constante de tiempo $\tau_{2}$ y ganancia unitaria. Esto nos permite obtener el instante de tiempo en el que la estimación del fallo cruza 

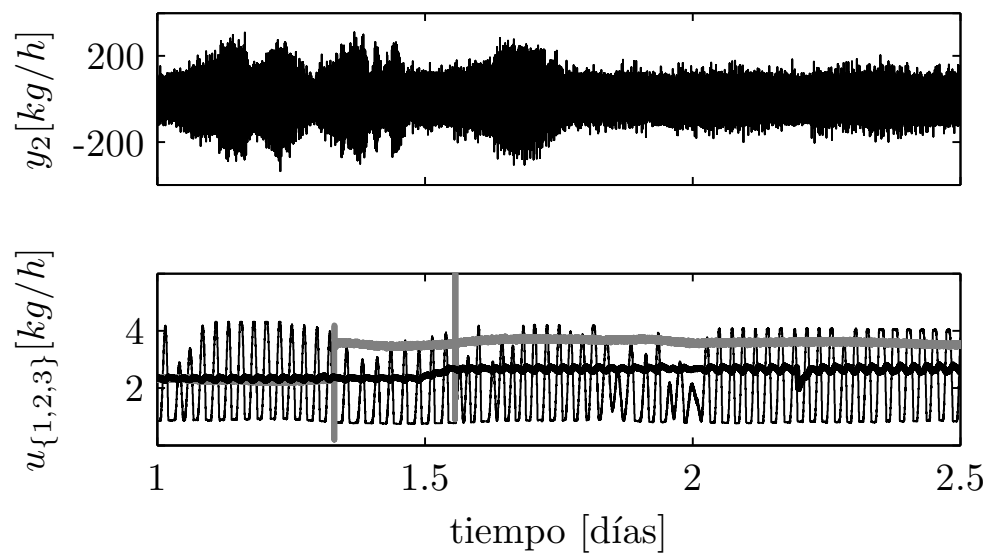

Figura 2: Entradas y salidas reales del tanque $2, T_{s}=1$.

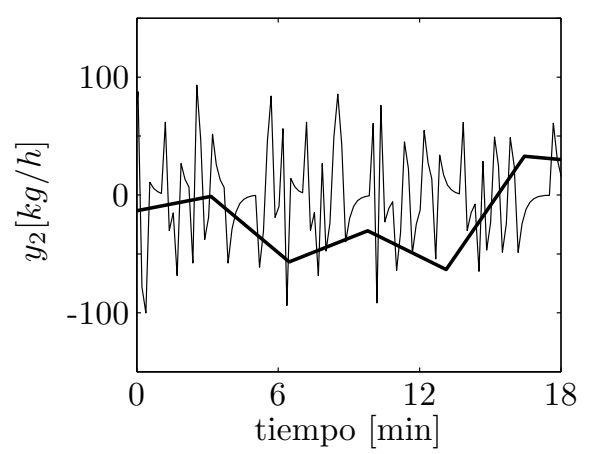

Figura 3: Detalle de la medición real $y_{2}$ para periodos de muestreo diferentes. (Claro: $T_{s}=1$, Oscuro: $T_{s}=20$ )
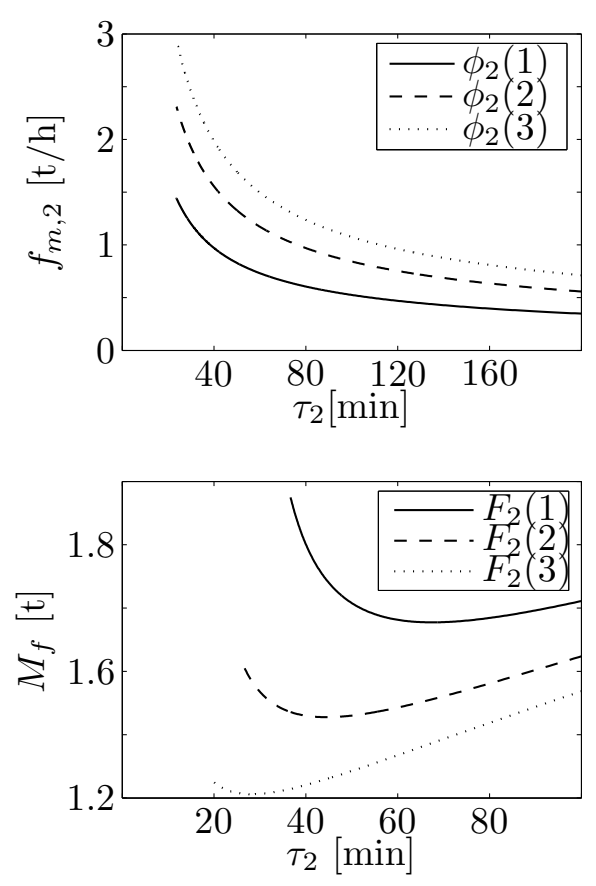

Figura 4: Compromiso de prestaciones para diferentes diseños. el umbral y, por lo tanto, la cantidad de masa que se ha perdido hasta la detección:

$$
M_{f}=-F \tau \log \left(1-\frac{f_{m}}{F}\right) .
$$

La parte inferior de la Figura 4 se representa esta masa para diferentes estimadores (con las constantes de tiempo indicadas el en eje horizontal, y con $\mathrm{FAR}=10^{-3}$ ) bajo tres fallos escalón de tamaños $F_{2}(1)=2 \mathrm{t} / \mathrm{h}, F_{2}(2)=3 \mathrm{t} / \mathrm{h}$, y $F_{2}(3)=5 \mathrm{t} / \mathrm{h}$. Dado un detector definido por $\tau_{2}$ y $f_{m, 2}$, es remarcable que una fuga mayor $F_{2}$ hace que el tiempo de detección ocurra más rápido de forma que se pierde menos masa durante la fuga. La masa fugada es infinita si el fallo $F_{2}$ es menor que el umbral $f_{m, 2}$, y alcanza un mínimo para cierto $\tau_{2}$. Como el fallo no se conoce a priori, esta característica no puede utilizarse para encontrar un detector óptimo (en el sentido de encontrar el detector que asegura una masa fugada mínima hasta la detección).

Para probar la bondad del enfoque, hemos alimentado el algoritmo (12) con datos reales para detectar fallos en el tanque 2. Hemos añadido una señal en forma de escalón de $3 \mathrm{t} / \mathrm{h}$ desde el instante $5 \mathrm{~h}$ en las mediciones de $m_{1}$, lo que representa una pérdida de esa cantidad en la tubería que va desde el caudalímetro hasta el tanque 2. Hemos probado el enfoque para diferentes diseños con tres valores diferentes de $f_{m, 2}$ y para un valor fijo de FAR de $10^{-3}$. El comportamiento del estimador y se puede ver en la Figura 5 (en oscuro). Se observa que en los periodos libres de fallo iniciales, la estimación tiene un pequeño sesgo debido a la densidad considerada en los caudalímetros, tal y como se explica en la ecuación (3). También se ha considerado datos simulados a partir de la generación de señales de caudal y obteniendo el volumen por integración, añadiendo también ruidos Gaussianos con la mayor varianza observada en los datos reales. En este caso, se tiene una estimación sin sesgo en los 

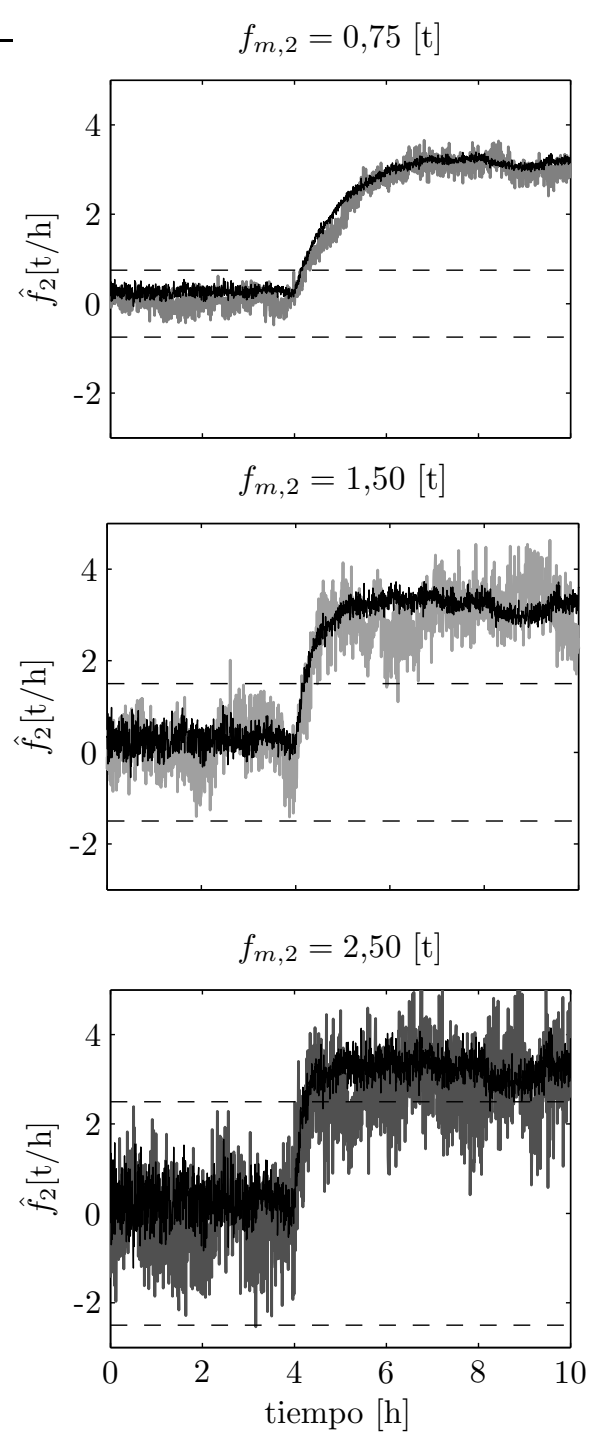

Figura 5: Comparación de las estimaciones y diagnóstico de diferentes diseños. (Oscuro: Datos industriales, Claro: Datos simulados.)

periodos libres de fallo que satisface con precisión el FAR diseñado.

\section{Conclusión}

En este trabajo se ha analizado el problema de la detección de fugas en redes de tuberías y tanques industriales, cuya masa y caudales se miden con sensores ruidosos. Se ha descrito y modelado el problema y se ha propuesta una estrategia de estimación. La estrategia modela las fugas como fallos aditivos y se propone el uso de un observador PI para su estimación. Este enfoque no requiere ninguna excitación específica para las señales y se pueden caracterizar las prestaciones obtenidas a pesar del nivel elevado de ruido de las señales disponibles. Estas prestaciones se han caracterizado por medio de los fallos mínimos diagnosticables, el ratio de falsas alarmas, y el ratio de convergencia ante fallos tipo escalón. Se ha mostrado la relación entre estos tres índices de desempeño para el ruido disponible en datos industriales reales. Finalmente, se ha simulado la presencia de fugas perturbando las mediciones en los caudalímetros y se ha estudiado su comportamiento. Se ha demostrado la validez del enfoque para fugas en tuberías que rodean cierto tanque.

\section{Agradecimientos}

Este trabajo ha sido financiado por la Universitat Jaume I de Castelló a través del proyecto con referencia P11B2015-42 2 y por el Ministerio de Economía y Competitividad a través del proyecto con referencia TEC2015-69155-R. También se ha financiado a través de la ayuda predocotoral FPU14/01592 del Ministerio de Educación, Cultura y Deporte. Los autores quieren mostrar su agradecimiento a UBE Corporation Europe, S.A.U. por su colaboración al compartir los datos industriales utilizados en el caso de estudio.

\section{Referencias}

[1] Boulos, Paul y Tom Altman: A graphtheoretic approach to explicit nonlinear pipe network optimization. Applied Mathematical Modelling, 15(9):459-466, 1991.

[2] Poulakis, Z, Dimitris Valougeorgis y Costas Papadimitriou: Leakage detection in water pipe networks using a Bayesian probabilistic framework. Probabilistic Engineering Mechanics, 18(4):315-327, 2003.

[3] Geiger, Gerhard, Thomas Werner y Drago Matko: Leak detection and locating - a survey. En PSIG Annual Meeting. Pipeline Simulation Interest Group, 2003.

[4] Murvay, Pal Stefan y Ioan Silea: A survey on gas leak detection and localization techniques. Journal of Loss Prevention in the Process Industries, 25(6):966-973, 2012.

[5] Verde, Cristina: Accommodation of multi-leak location in a pipeline. Control Engineering Practice, 13(8):1071-1078, 2005.

[6] Sun, Liang: Mathematical modeling of the flow in a pipeline with a leak. Mathematics and Computers in Simulation, 82(11):22532267, 2012.

[7] Andersen, Johannes H y Roger S Powell: Implicit state-estimation technique for water network monitoring. Urban Water, 2(2):123130, 2000. 
[8] Doorhy, James: Real-time pipeline leak detection and location using volume balancing. Pipeline \& Gas Journal, 238(2):65-66, 2011.

[9] Torres, Lizeth, Cristina Verde, Gildas Besançon y Omar González: High-gain observers for leak location in subterranean pipelines of liquefied petroleum gas. International Journal of Robust and Nonlinear Control, 24(6):1127-1141, 2014.

[10] Reddy, H Prashanth, Shankar Narasimhan, S Murty Bhallamudi y S Bairagi: Leak detection in gas pipeline networks using an efficient state estimator. Part II. Experimental and field evaluation. Computers \& Chemical Engineering, 35(4):662-670, 2011.

[11] Yin, Shen, Steven X Ding, Xiaochen Xie y Hao Luo: A review on basic data-driven approaches for industrial process monitoring. IEEE Transactions on Industrial Electronics, 61(11):6418-6428, 2014.

[12] Ding, Steven X: Model-based fault diagnosis techniques: design schemes, algorithms, and tools. Springer Science \& Business Media, 2008.

[13] Khedher, Atef, Kamel Benothman, Didier Maquin y Mohamed Benrejeb: State and sensor faults estimation via a proportional integral observer. En 6th International MultiConference on Systems, Signals and Devices, 2009., páginas 1-6. IEEE, 2009.

[14] Koenig, Damien: Unknown input proportional multiple-integral observer design for linear descriptor systems: application to state and fault estimation. IEEE Transactions on Automatic Control, 50(2):212-217, 2005. 